\title{
Viabilidade Espermática do Sêmen de Piracanjuba (Brycon orbignyanus) Resfriado a $4^{\circ} \mathrm{C}$ Luis David Solis Murgas ${ }^{1}$, Aléssio Batista Miliorini², Renan Toledo Franciscatto ${ }^{3}$, Alexandre Nízio Maria ${ }^{4}$
}

\begin{abstract}
RESUMO - Os efeitos de três meios diluidores (BTS+KCl - D1, BTS+2KCL - D2 e BTS+Citrato de Sódio - D3), considerando três diferentes tempos de resfriamento ( 0,72 e 144 horas), foram analizados no sêmen de Piracanjuba (Brycon orbignyanus) a $4^{\circ} \mathrm{C}$. Testou-se, ainda, a utilidade do meio diluidor BTS (Beltsville Thawing Solution), adicionado a citrato de sódio e cloreto de potássio, na manutenção da imobilidade dos espermatozóides e na conservação de características do sêmen. Resultados satisfatórios foram encontrados até 144 horas de resfriamento. A motilidade espermática média do sêmen in natura foi de $94 \pm 5,48 \%$, com duração média de 55,6 \pm 32,25 segundos (s). Para o sêmen diluído, as médias da motilidade e sua duração foram de $76,00 \pm 1,86 \%$ e $56,6 \pm 6,54 \mathrm{~s}, 77,00 \pm 1,86 \%$ e $54,6 \pm 6,54 \mathrm{~s}$, e 74,33 $\pm 1,86 \%$ e 75,0 $\pm 6,54 \mathrm{~s}$, respectivamente, para os diluidores D1, D2 e D3. Não houve diferenças significativas entre os diluidores, que apresentaram efeitos semelhantes nos diferentes tempos. As análises nos tempos 0 e 72 horas mostraram motilidades espermáticas superiores àquelas nas 144 horas, embora as durações da motilidade espermática não tenham diferido nesses tempos. A concentração espermática média do sêmen foi de $8,21 \pm 2,26 \mathrm{X} 10^{9} \mathrm{sptz} / \mathrm{mL}$. Algumas amostras evidenciaram motilidade espermática antes da ativação do sêmen (pré-ativação), principalmente a partir das 96 horas de resfriamento. O meio diluidor BTS pode ser recomendado para conservação de sêmen de piracanjuba (B. orbignyanus), desde que sua osmolaridade seja elevada.
\end{abstract}

Palavras-chave: Brycon orbignyanus, BTS, piracanjuba, resfriamento, sêmen

\section{Spermatic Viability of Piracanjuba (Brycon orbignyanus) Semen Cooled at $4^{\circ} \mathrm{C}$}

\begin{abstract}
The experiment was conducted in order to evaluate the effect of 3 diluents BTS $+\mathrm{KCl}-\mathrm{D} 1, \mathrm{BTS}+2 \mathrm{KCL}-$ D2 and BTS+Sodium Citrate - D3) and three different times (hour 0, 72 hours and 144 hours) in Piracanjuba (Brycon orbignyanus) semen at $4^{\circ} \mathrm{C}$. The assay aimed to test usefulness of BTS (Betsville Thawing Solution) diluent medium, added to Sodium citrate and Potassium clorate, in maintenance of spermatic immobility and of its conditions to conserve semen characteristics as spermatic motility rate and duration. Satisfactory results were found until at 144 hours of cooling for this specie. The mean spermatic motility in the in natura state was $94 \pm 5.48 \%$ with mean duration of $55.6 \pm 32.25$ seconds (s). For diluted semen, motility and duration means were $76.00 \pm 1.86 \%$ and $56.6 \pm 6.54 \mathrm{~s}, 77.00 \pm 1.86 \%$ and $54.6 \pm 6.54 \mathrm{~s}$, and $74.33 \pm 1.86 \%$ and $75.0 \pm 6.54 \mathrm{~s}$, respectivaly, for diluents D1, D2 and D3, in this order. Significant differences were not observed among diluents and they showed similar effects in at different times. At 0 and $72 \mathrm{~h}$ of analysis showed higher spermatic motilities than that ones at $144 \mathrm{~h}$, but durations of spermatic motility did not show differences in these times. The semen mean spermatic concentration was $8.21 \pm 2.26 \times 10^{9} \mathrm{sptz} / \mathrm{mL}$. Some samples showed spermatic motility before semen activation (foreactivation) principally after 96 hours of cooling. Thus, BTS medium diluent may be recommended for fish semen conservation, since its osmolarity is elevated.
\end{abstract}

Key Words: Brycon orbignyanus, BTS, cooling, piracanjuba, semen

\section{Introdução}

A busca constante pela otimização de processos técnicos de preservação de espermatozóides de peixes responde aos objetivos econômicos e ecológicos atuais. A preservação de sêmen a curto prazo consiste na manutenção de sua viabilidade por um período de horas ou dias, usualmente em temperaturas de refrigeração, sendo indicada para facilitar o manejo, por dispensar a presença do macho no ato da fecundação, e também para aumentar a eficiência da reprodução artificial nas estações de piscicultura.

\footnotetext{
${ }^{1}$ Médico Veterinário, Dr. Professor Adjunto do Departamento de Medicina Veterinária da UFLA (Ismurgas@ufla.br).

2 Aluno do curso de Graduação de Medicina Veterinária da UFLA e Bolsista da FAPEMIG, Convênio CEMIG/FAEPE/UFLA (abmiliorini@veterinaria.ufla.br).

3 Aluno do curso de Graduação de Medicina Veterinária da UFLA e Bolsista do CNPq, Convênio CEMIG/FAEPE/UFLA (renan@ufla.br).

${ }^{4}$ Aluno do curso de Graduação de Medicina Veterinária da UFLA e Estagiário do Convênio CEMIG/FAEPE/UFLA.
} 
A técnica é pouco dispendiosa e permite que o sêmen preservado esteja apto à fertilização de ovócitos de fêmeas induzidas hormonalmente, em vários intervalos de tempo, garantindo maior produtividade no processo de reprodução e o estudo das características do sêmen (Ciereszko \& Dabrowski, 1994), além de facilitar seu transporte (Davy \& Chouinard, 1980).

Os peixes de piracema têm tido sua reprodução e população negativamente afetadas pela construção de usinas hidrelétricas e modificação do curso natural dos rios pela presença de grandes lagos artificiais. As espécies da família Bryconidae foram significantemente prejudicadas pela construção de barragens nos rios e pelo desmatamento ciliar.

A piracanjuba (Brycon orbignyanus Valenciennes, 1849) é espécie reofílica encontrada na bacia do Paraná-Uruguai, sobretudo nos rios Grande e Paraná (Castagnolli, 1992). Possui excelente qualidade de carne e rápido crescimento, podendo atingir até 80 centímetros de comprimento corporal e 10 quilogramas de massa (Santos, 1981).

Os espermatozóides dos peixes são imóveis nas gônadas e somente apresentam motilidade quando em contato com a água ou outro meio suficientemente hiposmótico. Nas espécies dulcícolas, a motilidade espermática resulta, sobretudo, da abrupta redução da pressão osmótica do meio e pela diluição das altas concentrações de íons potássio do plasma seminal (Stoss \& Donaldson, 1982; Mutr \& Roberts, 1993).

Analisou-se, neste trabalho, a eficácia do BTS (Beltsville Thawing Solution-MINITUB ${ }^{\circledR}$ ), recomendado para a criopreservação do sêmen de suíno, na conservação a curto prazo do sêmen de piracanjuba, mediante a adição de solutos que proporcionem imobilidade espermática.

\section{Material e Métodos}

Foram utilizados cinco reprodutores de piracanjuba com peso corporal médio de $1,16 \mathrm{~kg}$, alocados em aquários com temperatura controlada em $28^{\circ} \mathrm{C}$. Os animais foram submetidos a tratamento hormonal com Extrato de Hipófise de Carpa (EHC). As hipofisações foram realizadas em duas doses com intervalo de doze horas, sendo a prévia $0,3 \mathrm{mg}$ de $\mathrm{EHC} / \mathrm{kg}$ de peso corporal e a aplicação efetiva $3,0 \mathrm{mg}$ de $\mathrm{EHC} / \mathrm{kg}$ de peso corporal. Antes da coleta, os animais foram contidos com o auxílio de uma toalha umedecida sendo a região do poro genital seca e limpa com papel. O critério de seleção dos espécimes foi baseado na liberação de esperma após leve pressão abdominal no sentido crânio-caudal (Woynarovich \& Horvath, 1989).

O sêmen foi coletado, aproximadamente, doze horas após a última dose de EHC, separadamente em tubos de ensaio graduados em mililitros, submersos em água a $28^{\circ} \mathrm{C}$ e protegidos da luz. Realizou-se exame macroscópico do sêmen no tubo coletor, observando-se seu aspecto, sua coloração e a ocorrência de contaminação por sangue, fezes ou urina. Procedeu-se à análise do sêmen in natura por meio de ativação com água destilada verificando sua motilidade e duração. A valorização da motilidade dos espermatozóides foi baseada na estimativa da porcentagem de células móveis no campo focalizado em aumento de 100X em microscópio óptico, segundo a escala arbitrária de 0 a $100 \%$ (Salisbury \& Vandemark, 1964). A duração (segundos) da motilidade espermática, considerada desde a mistura da água destilada até $10 \%$ de taxa de motilidade, foi mensurada com um cronômetro esportivo. O sêmen foi transportado em caixa térmica de isopor, com o ambiente de resfriamento gerado pela presença de gelo reciclável. A temperatura de transporte oscilou de 8 a $10^{\circ} \mathrm{C}$. Posteriormente, as amostras foram colocadas em refrigerador comum sob temperatura constante de $4^{\circ} \mathrm{C}$.

A determinação da concentração espermática foi realizada em câmara hematimétrica com uma alíquota de $0,001 \mathrm{~mL}$ de sêmen diluída em $1 \mathrm{~mL}$ de solução de formol citrato.

Para conservação do sêmen, utilizaram-se três diferentes diluidores:

I. Diluidor 1(D1) - BTS e $\mathrm{KCl}$ (quantidade igual de $\mathrm{KCl}$ que compõe o BTS)

II. Diluidor 2 (D2) - BTS e $\mathrm{KCl}$ (quantidade igual ao dobro de $\mathrm{KCl}$ que compõe o BTS)

III. Diluidor 3 (D3) - BTS e citrato de sódio (quantidade igual de citrato de sódio que compõe o BTS).

O sêmen foi diluído na proporção de 3:1 (uma parte de sêmen para três de diluidor) em tubos de ensaio, de forma a minimizar o choque osmótico, e mantido nos tubos de ensaio com ar atmosférico (Marques, 2001). Na avaliação inicial-tempo 1 (0 h), analisou-se a imotilidade das células espermáticas das amostras diluídas e a in natura. O mesmo processo foi adequado às análises das amostras nos tempos 2 (72 h) e 3 (144 h).

R. Bras. Zootec., v.33, n.6, p.1361-1365, 2004 
Utilizou-se delineamento inteiramente ao acaso, com cinco repetições e tratamentos dispostos em estrutura fatorial 3 x 3 (diluidores x tempos de resfriamento).

Os resultados obtidos foram submetidos à análise estatística por meio do Sistema de Análise de Variância (SISVAR - Ferreira, 1999) e as médias foram comparadas pelo teste Tukey.

\section{Resultados e Discussão}

De acordo com os dados obtidos na avaliação do sêmen in natura a $28^{\circ} \mathrm{C}$, os resultados das estimativas da motilidade espermática e sua duração oscilaram de 90 a $100 \%$, e de 36 a 113 segundos, com média de $94,00 \pm 5,48 \%$ e $55,60 \pm 32,25$ segundos, respectivamente. Kabeya et al. (1998), para a mesma temperatura, obteve com piapara (Leporinus obtusidens) médias de $81,07 \%$ e de 216,59 s para motilidade dos espermatozóides. Cruz (2001), trabalhando com curimbatá (Prochilodus lineatus), verificou taxa e duração para a motilidade espermática do sêmen fresco de $90,00 \pm 0,00 \%$ e $111,40 \pm 86,50 \mathrm{~s}$, respectivamente. Os resultados da análise de variância comprovam que não houve diferença significativa dos diluidores em relação à motilidade espermática do sêmen resfriado de piracanjuba dentro dos tempos considerados $(\mathrm{P}>0,05)$, embora os diluidores com adição de $\mathrm{KCl}$ tenham apresentado dados superiores (Tabela 1). Entretanto, verificou-se que, nos diferentes tempos, as motilidades médias do sêmen analisado foram significativamente diferentes $(\mathrm{P}<0,01)$, ou seja, o sêmen de piracanjuba resfriado a $4^{\circ} \mathrm{C}$ apresentou qualidade superior até 72 horas de resfriamento.
Em peixes da mesma espécie, Marques (2001) constatou taxas de motilidade espermática de 50,00 \pm $0,00 \%$, em saco com oxigênio, e $25,00 \pm 7,00 \%$, em tubo de ensaio, após 12 horas de resfriamento com gelo ou termogel. Murgas et al. (1998) encontraram taxa de $60 \%$ de motilidade do sêmen criopreservado, usando o diluidor água de côco. Santana (1998) relata para o pacu (Piaractus mesopotamicus) motilidade média de $23,75 \pm 15,44 \%$ do sêmen, após 24 horas de resfriamento a $4^{\circ} \mathrm{C}$. Na presença do diluidor D3, foram observadas formações de grumos que incluíam a maioria dos espermatozóides.

A taxa média de motilidade espermática foi de $58,33 \pm 4,57 \%$ às 72 horas de resfriamento a $4^{\circ} \mathrm{C}$ do sêmen de pacu (Piaractus mesopotamicus), sob ativação de bicarbonato de sódio (1\%) e diluição do sêmen com BTS e adicionado ao dimetilsulfóxido (DMSO), na concentração de $10 \%$. Franciscatto et al. (2002) utilizaram, em vez de DMSO, citrato de sódio e iodeto de potássio no resfriamento do sêmen de curimba (Prochilodus lineatus) e obtiveram resultados médios de 43,60 $\pm 3,92 \%$ de motilidade média até 168 horas após o início do resfriamento àquela mesma temperatura.

Os resultados da análise de variância ainda evidenciam que os diluidores continuaram apresentando efeitos semelhantes nos diferentes tempos quanto à duração da motilidade $(\mathrm{P}>0,05)$ (Tabela 2), uma vez que não houve diferença significativa entre as durações da motilidade observadas $(\mathrm{P}>0,05)$, indicando conservação da duração da motilidade espermática até as 144 horas de resfriamento a $4^{\circ} \mathrm{C}$ do sêmen de piracanjuba.

Tabela 1 - Taxas médias para a motilidade espermática, durante o período de 144 horas, para o sêmen de piracanjuba (Brycon orbignyanus) resfriado a $4^{\circ} \mathrm{C}$

Tabel 1 - Mean motility spermatic rates during 144 hours of piracanjuba (Brycon orbignyanus) semen cooling at $4^{\circ} \mathrm{C}$

\begin{tabular}{ccccc}
\hline \multirow{2}{*}{$\begin{array}{c}\text { Diluidores } \\
\text { Diluters }\end{array}$} & \multicolumn{3}{c}{$\begin{array}{c}\text { Tempos de resfriamento }(\mathrm{h}) \\
\text { Cooling times }(h)\end{array}$} & $\begin{array}{c}\text { Médias } \\
\text { Means }\end{array}$ \\
\cline { 2 - 4 } & 0 & 72 & 144 & \\
\hline BTS + KCl & $84,00 \pm 3,22$ & $79,00 \pm 3,22$ & $65,00 \pm 3,22$ & $76,00 \pm 1,86^{3}$ \\
BTS + 2KCl & $83,00 \pm 3,22$ & $82,00 \pm 3,22$ & $66,00 \pm 3,22$ & $77,00 \pm 1,86^{3}$ \\
BTS + CITRATO & $86,00 \pm 3,22$ & $80,00 \pm 3,22$ & $57,00 \pm 3,22$ & $74,33 \pm 1,86^{3}$ \\
Médias & $82,00 \pm 1,86^{1}$ & $80,33 \pm 1,86^{1}$ & $62,67 \pm 1,86^{2}$ & $75,00 \pm 1,07$ \\
Means & & & & \\
\hline
\end{tabular}

\footnotetext{
1,2 Médias seguidas de mesmo número, nas linhas, não diferem $(P>0,05)$ entre si.

3 Médias não diferem $(P>0,05)$ entre si.

1,2 Means followed by similar numbers, within a row, are not different $(P>.05)$ by Tukey test.

3 Means are not different $(P>.05)$ by Tukey test.
}

\section{R. Bras. Zootec., v.33, n.6, p.1361-1365, 2004}


Tabela 2 - Durações (s) médias para a motilidade espermática, durante o período de 144 horas, para o sêmen de piracanjuba (Brycon orbignyanus) resfriado a $4^{\circ} \mathrm{C}$

Tabel 2 - Mean motility spermatic durations during 144 hours of piracanjuba (Brycon orbignyanus) semen cooling at $4^{\circ} \mathrm{C}$

\begin{tabular}{ccccc}
\hline \multirow{2}{*}{$\begin{array}{c}\text { Diluidores } \\
\text { Diluters }\end{array}$} & \multicolumn{3}{c}{$\begin{array}{c}\text { Tempos de resfriamento (h) } \\
\text { Cooling times }(h)\end{array}$} & $\begin{array}{c}\text { Médias } \\
\text { Means }\end{array}$ \\
\cline { 2 - 4 } & 0 & 72 & 144 \\
\hline BTS + KCl & $60,40 \pm 11,33$ & $56,40 \pm 11,33$ & $53,00 \pm 11,33$ & $56,00 \pm 6,54^{2}$ \\
BTS + 2KCl & $63,20 \pm 11,33$ & $49,40 \pm 11,33$ & $51,20 \pm 11,33$ & $54,60 \pm 6,54^{2}$ \\
BTS + CITRATO & $79,00 \pm 11,33$ & $86,60 \pm 11,33$ & $59,40 \pm 11,33$ & $75,00 \pm 6,54^{2}$ \\
Médias & $67,53 \pm 6,54^{1}$ & $64,13 \pm 6,54^{1}$ & $54,53 \pm 6,54^{1}$ & $62,06 \pm 3,78$ \\
Means & & & & \\
\hline
\end{tabular}

${ }^{1}$ Médias não diferem $(P>0,05)$ entre si.

${ }^{2}$ Médias não diferem $(P>0,05)$ entre si.

${ }^{1}$ Means are not different $(P>05)$ by Tukey test.

${ }^{2}$ Means are not different $(P>.05)$ by Tukey test.

Marques (2001) verificou duração média da motilidade espermática para piracanjuba de $64,00 \pm 2,83$ s, para sêmen preservado em saco com oxigênio, e de $59,00 \pm 5,66 \mathrm{~s}$, para sêmen preservado em tubo de ensaio, próxima à encontrada neste trabalho. Murgas et al. (1998), obtiveram duração média de $125 \mathrm{~s}$ para a motilidade do sêmen de piracanjuba, após congelamento com o diluidor água de côco, superior à deste estudo. Os valores descritos por Silva et al. (1998), para duração da motilidade de sêmen de curimba ( $P$. lineatus) e piaparas (Leporinus elongatus e L. obtusidens), após 18 horas de resfriamento, foram de 54, 0 e $76 \mathrm{~s}$ para $P$. lineatus, $L$ elongatus e L. obtusidens, respectivamente.

Os valores registrados por Bedore (1999) foram de $10,00 \pm 4,30 \times 10^{9}$ células espermáticas (sptz) por mililitro, pouco superior aos $8,21 \pm 2,26 \times 10^{9} \mathrm{sptz} / \mathrm{mL}$ aqui encontrados. Esse mesmo autor contou 36,6 $\pm 16,5$ X $10^{9} \mathrm{sptz} / \mathrm{mL}$ em pacu-caranha (P. mesopotamicus), enquanto Fogli da Silveira et al. (1990) constataram valores de 28,07 $\pm 8,20 \times 10^{9} \mathrm{sptz} / \mathrm{mL}$. Trabalhando com dourado (Salminus maxillosus), Godinho \& Cóser (1995) contaram 4,30 a $10,80 \times 10^{9} \mathrm{sptz} / \mathrm{mL}$; com piapara (L. elongatus), 7,00 a 16,00 X $10^{9} \mathrm{sptz} / \mathrm{mL}$; e com curimbatá-pacu (Prochilodus marggravi), 19,20 a 26,60 X $10^{9} \mathrm{sptz} / \mathrm{mL}$. Todos os valores de concentração espermática citados advêm de animais também submetidos a tratamento hormonal.

\section{Conclusões}

O meio diluidor BTS pode ser usado para o sêmen de piracanjuba, desde que sejam adicionados solutos adequados em quantidades apropriadas para gerar um meio osmoticamente favorável à inativação da motilidade dos espermatozóides.

O sêmen de piracanjuba apresenta boa viabilidade após 144 horas de resfriamento, a $4^{\circ} \mathrm{C}$, quando diluído em BTS e Citrato de sódio ou $\mathrm{KCl}$.

Mais pesquisas devem ser realizadas com o BTS a fim de se corrigir seu efeito pré-ativador, e aumentar o período de conservação do sêmen da piracanjuba.

\section{Agradecimento}

À Companhia Energética de Minas Gerais CEMIG, pela fundamental colaboração na realização deste e de outros inúmeros trabalhos ligados à piscicultura, primando conosco pela preservação da fauna de Piracema e do ecossistema aquático.

\section{Literatura Citada}

BEDORE, A.G. Características criopreservação do sêmen de pacu-caranha (Piaractus mesopotamicus) e de piracanjuba (Brycon orbignyanus). Belo Horizonte: Universidade Federal de Minas Gerais, 1999. 53p. Dissertação (Mestrado em Biologia Celular) - Universidade Federal de Minas Gerais, 1999.

CAStagnolli, N. Criação de peixes de água doce. Jaboticabal: FUNEP. 1992. 189p.

CIEREZKO, A.; DABROWSKI, K. Relationship between biochemical constituents of fish semen and fertility: the effect of short-term storage. Fish Physiology and Biochemistry, v.12, n.5, p.357-367, 1994.

CRUZ, V.L.B. Criopreservação de sêmen de curimbatá (Prochilodus lineatus). Belo Horizonte: Pontifícia Universidade Católica de Minas Gerais, 2001.59p. Dissertação (Mestrado em Zoologia de Vertebrados) - Pontifícia Universidade Católica de Minas Gerais, 2001.

DAVY, F.B.; CHOUINARD, A. Induced fish breeding in south est asia: Ottawa: 1980. 48p. (Report of a work shop hekd in singapore). 
FERREIRA, D.F. Sistema de análise de variância - SISVAR - DEX/UFLA, ver 4.0 (Build 34). 1999.

FOGLI DA SILVEIRA, W.F.; KAVAMOTO, E.T.; CESTARELLI, M.A. et al. Avaliação espermática, preservação criogênica e fertilidade do sêmen de pacu, Piaractus mesopotamicus (HOLMBERG, 1887), proveniente da reprodução induzida. Instituto Biológico de Pesca, v.17 (único), p.1-13, 1990.

FRANCISCATTO, R.T.; MURGAS, L.D.S.; MILIORINI, A. B. et al. Qualidade do sêmen de curimba (Prochilodus lineatus) e taxa de fertilidade após resfriamento a $4^{\circ} \mathrm{C}$. Revista Brasileira de Reprodução Animal, v.26, n.3, 213-215, 2002.

GODINHO, H.P.; CÓSER, A.M.L. Bases morfofuncionais da espermatogênese e criopreservação de sêmen de peixes. In: CONGRESSO BRASILEIRO DE REPRODUÇÃO ANIMAL, 11., 1995, Belo Horizonte. Anais... Belo Horizonte: Colégio Brasileiro de Reprodução Animal, 1995. p.16-25.

KABEYA, D.M.; SILVA, M.O.B.; MELLO, C.B.M. et al. Avaliação quantitativa do sêmen in natura "Piaparas" (Leporinus obtusidens e L. elongatus), capturadas no rio Grande à jusante da UHE em Itutinga-MG. Resumo de aqüicultura Brasil' 98, 1998. 293p.

MARQUES, S. Preservação a curto prazo do sêmen de teleósteos neotropicais de água doce. Belo Horizonte: Pontifícia Universidade Católica de Minas Gerais, 2001, 81p. Dissertação (Mestrado em Zoologia) - Pontifícia Universidade Católica de Minas Gerais (PUC-MG), 2001.

MILIORINI, A.B.; MURGAS, L.D.S.; VIVEIROS; A.T.M. et al. Resfriamento do sêmen de pacu (Piaractus mesopotamicus) a $4^{\circ} \mathrm{C}$, utilizando diferentes concentrações de dimetilsulfóxido. Revista Brasileira de Reprodução Animal, v.26, n.3, 209-211, 2002.

MURGAS, L.D.S. Calidad seminal del pez piracanjuba (Brycon orbignyanus) post-descongelación. Anales de Veterinaria de Murcia, v.17, p.8-16, 1998.
MUTR, J.F.; ROBERTS, R.J. Recents advances en aquacuture IV. Cambridge: Blackwell Scientific Publications, 1993. p.15-18.

SALISBURY, G.W.; VANDEMARK, N.L. Fisiologia de la reproducción e inseminación artificial de los bóvidos. Zaragoza: Acribia, 1964. 707p.

SANTANA, G.M. Avaliação do sêmen de pacu (Piaractus mesopotamicus) após 6,18 e 24 horas de resfriamento à temperatura de $4^{\circ} \mathrm{C}$. In: CONGRESSO DE INICIAÇÃO CIENTÍFICA DA UFLA - CICESAL, 9., 1998, Lavras. Resumos... Lavras: Universidade Federal de Lavras, 1998. $272 p$.

SANTOS, E. Peixes de água doce. Belo Horizonte: Itatiaia, 1981. v.2, p.58-60.

SILVA, M.O.B.; MURGAS, L.D.S.; MELLO, C.B.M. et al. Avaliação do sêmen de "Curimba" (Prochilodus lineatus) e "Piaparas" (Leporinus obtusidens e L. elongatus) após 6, 18 e 24 horas de resfriamento à temperatura de $4^{\circ} \mathrm{C}$. In: AQÜICULTURA BRASIL'98, 1998, Recife. Resumos... Recife: 1998.

STOSS, J.; DONALDSON, E.M. Preservation of fish gametes. In: INTERNATIONAL SIMPOSIUM ON REPRODUCTIVE PHISIOLOGY OF FISH, 1982, Wageningen. Anais... Wageningen: 1982. p.114-122.

WOYNORAVICH, E.; HONORÁTH, L. A propagação artificial de peixes de águas tropicais. Brasília: CODEVASF, 1989. 225p. (Manual de extensão) 